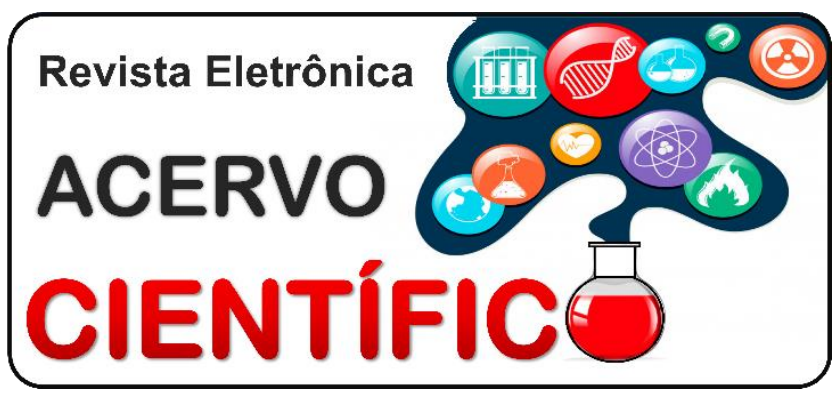

\section{REVISÃO BIBLIOGRÁFICA}

Recebido em: 1/2021

Aceito em: 1/2021

Publicado em: 2/2021

\title{
Epidemiologia e fatores de risco associados às neoplasias gástricas: uma revisão de literatura
}

\author{
Epidemiology and risk factors associated with gastric neoplasms: a literature review \\ Epidemiología y factores de riesgo asociados a neoplasias gástricas: una revisión de la \\ literatura
}

\begin{abstract}
Victoria Haydée Deusdedith Neves ${ }^{1 *}$, David Martins da Silva Mello ${ }^{1}$, Manuela Azevedo Vieira ${ }^{1}$, Ingrid Cristiane Pereira Gomes².
\end{abstract}

\begin{abstract}
Resumo: Este artigo buscou analisar o perfil epidemiológico e os fatores de risco mais relevantes relacionados às neoplasias gástricas. O estudo é uma revisão da literatura atual e foram inclusos 18 artigos da plataforma PUBMED, utilizando os seguintes descritores: stomach neoplasms, risk factors, epidemiology. As pesquisas epidemiológicas afirmam que aproximadamente 1 milhão de casos novos sujam a cada ano e colocam esta afecção como a terceira causa de morte por câncer no mundo. Os fatores de risco mais prevalentes foram idade avançada, sexo masculino, infecção por Helicobacter pylori, tabagismo, consumo de alimentos com alto teor de sódio e ingesta de bebidas alcoólicas. Deste modo, conclui-se que as neoplasias gástricas se constituem uma entidade nosológica de epidemiologia alarmante e decorre da interação de múltiplos fatores de risco, sejam eles modificáveis ou relacionados aos hábitos de vida e, por isso, faz-se essencial reconhecê-los, a fim de prevenir, ou ao menos, diagnosticar precocemente tal condição clínica.
\end{abstract}

Palavras-chave: Neoplasias gástricas, Fatores de risco, Epidemiologia.

\begin{abstract}
This article proposes de analyze the epidemiological profile and the most relavant risk factors related to gastric neoplasms. This study is a review of the current literature and 18 articles from PUBMED were included, by using the keywords: stomach neoplasms, risk factors, epidemiology. The epidemiological research claim that approximately 1 million new cases arise each year and put this disease the third cause of death by cancer in the world. The most prevalent risk factors were advanced age, male sex, Helicobacter pylori infection, smoking, intake of salty foods and alcohol consumption. Therefore, it is possible to conclude that the gastric neoplasms constitutes a disease with alarming epidemiology e stems from the interaction between multiple factors, whether modifiable or related to lifestyle habits; so, it is essential those are recognized, in order to prevent, or at least make an early diagnosis, of this clinical condition.
\end{abstract}

Keywords: Gastric neoplasms, Risk factors, Epidemiology.

Resumen: Este artículo buscó analizar el perfil epidemiológico y los factores de riesgo más relevantes relacionados con las neoplasias gástricas. El estudio es una revisión de la literatura actual y se incluyeron 18 artículos de la plataforma PUBMED, utilizando los siguientes descriptores: neoplasias estomacales, factores

\footnotetext{
1 Universidade Tiradentes (UNIT), Aracaju - SE. *E-mail: victoriahdneves@outlook.com

2 Universidade Federal de Sergipe (UFS), São Cristóvão - SE.
} 
de riesgo, epidemiología. La investigación epidemiológica afirma que aproximadamente 1 millón de casos nuevos se ensucian cada año y colocan esta condición como la tercera causa principal de muerte por cáncer en el mundo. Los factores de riesgo más prevalentes fueron la vejez, el sexo masculino, la infección por Helicobacter pylori, el tabaquismo, el consumo de alimentos con alto contenido de sodio y la ingestión de bebidas alcohólicas. Por lo tanto, se concluye que las neoplasias gástricas constituyen una entidad nosológica de epidemiología alarmante y son el resultado de la interacción de múltiples factores de riesgo, ya sean modificables o relacionados con los hábitos de vida y, por lo tanto, es esencial reconocerlos, para prevenir, o al menos, diagnosticar dicha condición clínica temprano.

Palabras clave: Neoplasias gástricas, Factores de riesgo, Epidemiología.

\section{INTRODUÇÃO}

O câncer gástrico constitui-se o quarto tipo mais comum de câncer nos homens e o quinto nas mulheres, colocando-o como responsável por 6,8\% das mortes por câncer no mundo (FANG X, et al., 2015). Em números absolutos, estima-se que aproximadamente 990.000 indivíduos são diagnosticados com câncer gástrico a cada ano e destes, 738.000 morrem pela doença (CAVATORTA O et al., 2018).

No tangente ao sexo, é uma desordem mais comum em homens, numa proporção de aproximadamente 3:1, e faz-se mais comum em indivíduos acima de 55 anos (CAVATORTA O, et al., 2018; SONNENBERG WR, 2017).

De forma geral, nota-se tendência atual à redução da incidência do câncer de estômago na maior parte do mundo, no entanto, as estatísticas concernentes ao tumor de cárdia continuam estáveis ou com predisposição ao aumento (CAVATORTA O, et al., 2018). Esta dissemelhança provavelmente provém dos diferentes fatores de risco relacionados ao desenvolvimento de tais neoplasias. Os tumores de cárdia, por exemplo, não possuem a infecção pelo Helicobacter pylori como fator de risco, mas sim a obesidade e a doença do refluxo gastroesofágico (DRGE), cujas incidências encontram-se em exponencial crescimento (CAVATORTA O, et al., 2018). Outrossim, múltiplas pesquisas atestam o impacto dos hábitos dietéticos e comportamentais no desenvolvimento de tal enfermidade (FANG X, et al., 2015).

No Brasil, o câncer de estômago ocupa o quarto lugar em prevalência de câncer entre os homens e o quinto entre as mulheres e, devido ao diagnóstico tardio em aproximadamente $75 \%$ dos casos, a taxa de sobrevivência decaiu nos últimos anos, em contraste à tendência mundial. Ainda são notadas diferenças estatísticas significativas entre as regiões, com maiores taxas de mortalidade do Norte e Nordeste do País, justificadas pelas disparidades socioeconômicas e de acesso à saúde (BRAGA L, et al., 2019).

As neoplasias de estômago possuem curso inicialmente silencioso, de forma que o quadro clínico clássico manifestar-se-á apenas em estágios mais avançados da doença. Dentre os sinais e sintomas mais comuns, destaca-se a perda ponderal, vista em 70 a $80 \%$ dos pacientes. Ademais, pode haver plenitude gástrica, anorexia, dispepsia e, nos casos de tumores de junção gastroesofágica, disfagia. O exame clínico também é passível de alterações, revelando massa epigástrica, hepatomegalia, icterícia, ascite e ainda achados compatíveis com doença metastática, tais como linfonodos de Virshow e da irmã Maria José e o sinal da prateleira de Blumer (SONNENBERG WR, 2017).

Dentre os métodos diagnósticos, o mais empregado é a endoscopia digestiva alta com biópsia. O estadiamento, por sua vez, é realizado através da tomografia computadorizada (TC) de tórax, abdome e pelve (SONNENBERG WR, 2017). A neoplasia de estômago possui tratamento complexo, vez que depende da extensão e localização do tumor, podendo ser empregadas modalidades cirúrgicas, radioterápicas e/ou quimioterápicas. No que concerne ao prognóstico, este tende a ser reservado, dado que, devido à clínica inicialmente sutil, o diagnóstico de tal condição mórbida faz-se majoritariamente tardio (SONNENBERG WR, 2017).

Esta revisão bibliográfica é fomentada pela observação da alta incidência das neoplasias gástricas, a despeito do conhecimento de seus principais fatores de risco, e por sua elevada morbimortalidade, 
características que a tornam um relevante problema de saúde pública. O principal objetivo desta pesquisa foi identificar as características epidemiológicas prevalecentes das neoplasias gástricas, assim como os principais fatores de risco a ela associados.

\section{MÉTODOS}

O presente artigo é revisão integrativa da literatura com análise e síntese dos resultados observados. A revisão em questão foi guiada pelo seguinte questionamento "Qual o perfil epidemiológico das neoplasias gástricas e quais são os principais fatores de risco associados a esta enfermidade?".

Foi realizada uma busca mediante base de dados do Medical Literature Analysis and Retrieval System Online (MEDLINE), por meio do mecanismo de busca Public/ Publisher MEDLINE (PubMed). Os descritores de interesse, buscados através da ferramenta Medical Subject Headings (MeSH), foram: stomach neoplasms, risk factors e epidemiology, os quais foram escolhidos com base na observação das principais características da temática abordadas em estudos prévios. A estratégia de busca foi "((stomach neoplasms) AND (risk factors)) AND (epidemiology)".

Os critérios de inclusão utilizados para a seleção dos artigos foram: artigos presentes na base de dados selecionada, nas línguas português e/ou inglês, e que foram publicados nos últimos 5 anos. Os critérios de exclusão, por sua vez foram os artigos em línguas diferentes das utilizadas pelo critério de inclusão, os incompletos e os que ultrapassavam a data limite de 5 anos (Figura 1). A busca foi realizada mediante acesso online dos artigos relevantes durante os meses de maio a agosto de 2020, com posterior análise do material coletado para a reunião das informações mais relevantes de cada publicação.

\section{RESULTADOS E DISCUSSÃO}

$\mathrm{Na}$ presente revisão de literatura foram inclusos 20 artigos. Durante a busca foram encontrados 5.744 resultados, com descarte de parte deles por não atenderem aos critérios propostos. Os artigos selecionados e utilizados na confecção do presente artigo encontram-se dispostos no Quadro 1.

Figura 1 - Fluxograma de critérios de inclusão e exclusão da pesquisa.

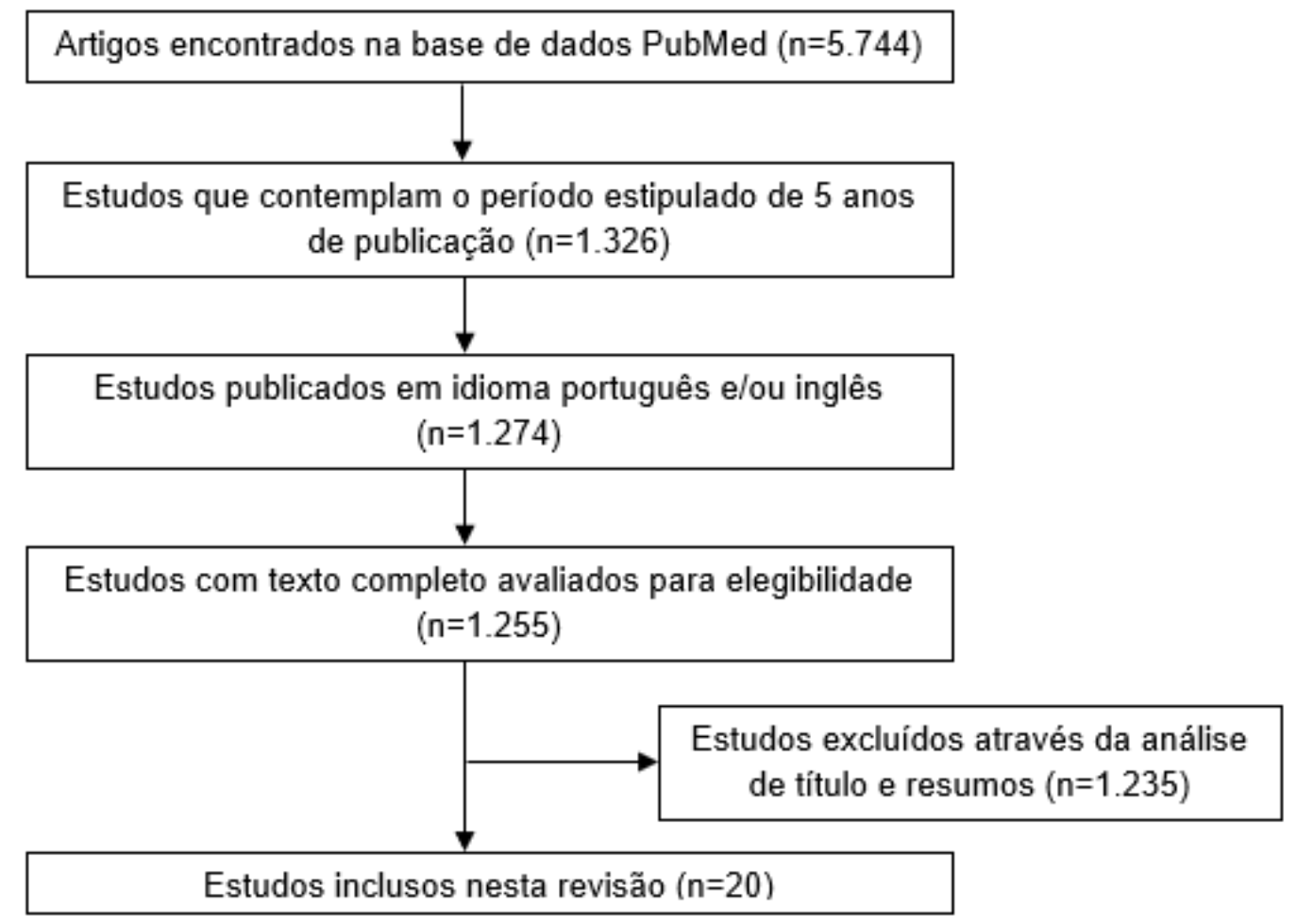

Fonte: Neves VH, et al., 2020. 
Percebe-se uma tendência mundial à redução da incidência das neoplasias gástricas, numa taxa de aproximadamente $2,5 \%$ ao ano (VENNEMAN K, et al., 2018). Tal decréscimo pode ser atribuído, majoritariamente, a dois grandes fatores: 1) melhor preservação dos alimentos, principalmente das frutas e vegetais e 2) redução da incidência do maior fator de risco: a infecção por $H$. pylori (BALAKRISHNAN M, et al., 2017). Não obstante, estas neoplasias ainda são fontes de grande morbimortalidade em todo o mundo (VENNEMAN K, et al., 2018).

A neoplasia de estômago é a terceira causa de morte por câncer no mundo em ambos os sexos VENERITO $M$, et al., 2018). Tais estatísticas resultam do diagnóstico usualmente tardio da doença, tendo em vista o quadro clínico inicialmente oligossintomático e a dificuldade de identificação de lesões sutis por métodos endoscópicos (CAVATORTA O, et al., 2018). Na América Latina, a mortalidade por câncer gástrico é alta, sendo isto retrato do baixo número de diagnósticos feitos em estágios precoces, a exemplo do México, local no qual apenas $2,5 \%$ dos casos da doença são diagnosticados ainda nesta fase (RUíZ-GARGIA E, et al., 2017). A taxa de sobrevida em cinco anos depende do estágio evolutivo da neoplasia: nos cânceres precoces aproxima-se de $90 \%$, contra $20 \%$ nos casos avançados (CHEN P, et al., 2019).

Geograficamente, percebe-se maior incidência na Ásia Oriental, Europa Central e Oriental e América Central e do Sul. Destes, a primeira constitui-se a região mais afetada e, em 2012, a China, Japão e Coreia, juntos, concentraram $60 \%$ dos novos casos da doença. Apesar da elevada incidência, a mortalidade é significativamente menor que na América do Norte, fato que provavelmente decorre do maior número de diagnósticos precoces naquela região, apesar de diferenças biológicas do tumor também contribuírem para a estatística (BALAKRISHNAN M, et al., 2017).

Curado M, et al. (2019) observaram que, no Brasil, a maior incidência de adenocarcinoma gástrico é na cidade de Belém. Outrossim, a pesquisa em questão verificou tendência de redução da incidência em São Paulo, em ambos sexos, e em Fortaleza, entre os homens. Curado M, et al. (2019) ainda constataram que, de forma geral, a mortalidade por neoplasia de estômago está diminuindo no Brasil, embora a incidência de tal doença tenha aumentado em Belém e Fortaleza, dado que revela o reflexo do acesso desigual aos serviços de saúde entre as regiões brasileiras.

As neoplasias gástricas podem ser divididas segundo critérios anatômicos, histopatológicos ou de evolução. Anatomicamente, pode-se dividi-las em dois grandes grupos: os tumores cárdicos e os não cárdicos. Esta classificação faz-se relevante pois cada um deles traz consigo características clinicoepidemiológicas próprias (CAVATORTA O, et al., 2018). Do ponto de vista histológico, os tumores podem ser classificados em intestinal ou difuso: enquanto o primeiro possui forte associação com a infecção por $H$. pylori, o segundo costuma originar-se de uma mucosa previamente normal, relacionando-se à obesidade, status socioeconômico alto e tipo sanguíneo A (HIRABAYASHI M, et al., 2019). Quanto à evolução, tais neoplasias podem ser precoces, quando limitadas à mucosa e/ ou submucosa e sem invasão linfonodal, e avançadas, caracterizadas pela invasão da muscular da mucosa ou planos mais profundos (CAVATORTA O, et al., 2018).

O adenocarcinoma é o tipo mais comum das neoplasias gástricas, responsável por 90-95\% dos casos. Outros tipos menos comuns são os linfomas, leiomiossarcomas, tumores neuroendócrinos e tumor estromal gastrointestinal (CAVATORTA O, et al., 2018). Em sua pesquisa, Cavatorta O, et al. (2018) dividiu os fatores de risco em não-modificáveis e modificáveis. No primeiro grupo encontram-se idade avançada, sexo masculino, etnia, história familiar e presença de síndromes hereditárias predisponentes. O segundo, por sua vez, é formado majoritariamente por condições comportamentais, tais como tabagismo, consumo alcoólico, exposição à radiação e infecção pela $H$. pylori.

Os fatores de risco gerais observados na revisão realizada por Cavatorta O, et al. (2018) foram idade avançada, sexo masculino, tabagismo, etnia branca e exposição à radiação. No que concerne aos tumores de cárdia, os mais relevantes foram a obesidade, principalmente quando $I M C>40$, e a doença do refluxo gastroesofágico (DRGE), que acarretaram aumento do risco de 3 e 4 vezes, respectivamente. Os tumores gástricos não-cárdicos, por sua vez, mostraram-se mais comuns na concomitância à infeção pelo $H$. pylorie também relacionado ao consumo de comidas de alto teor sódico. 
A infecção por $H$. pylori é considerada o maior fator de risco para a ocorrência de neoplasias gástricas (VENERITO M, et al., 2018). As vias pelas quais tal condição predispõe à doença em questão ainda não foram completamente elucidadas, mas sabe-se que se associa ao desenvolvimento de gastrite atrófica e metaplasia intestinal, importantes lesões pré-neoplásicas. Pesquisas ressaltam, no entanto, que o efeito carcinogênico de tal microrganismo é insuficiente para causar neoplasias na ausência de outros fatores e, por isso, a progressão carcinogênica é dependente de variáveis como cepa bacteriana, resposta imune do hospedeiro e exposição a fatores ambientais (VENNEMAN K, et al., 2018; BALAKRISHNAN M, et al., 2017).

Dentre os fatores de virulência do microrganismo, o gene CagA merece destaque, visto que este interage com determinadas proteínas do hospedeiro, induzindo alterações morfológicas que podem predispor alterações epigenéticas relacionadas à carcinogênese (POOROLAJAL J, et al., 2020).

Venneman K, et al. (2018), em revisão sistemática, concluiu que as maiores taxas de infecção por H. pylori foram encontradas na população mais velha e, em relação ao sexo, não foram encontradas diferenças significativas. Corroborando com tal estudo, Lee Y-C, et al. (2016), mediante meta-análise de 24 trabalhos, observaram que os indivíduos nos quais a $H$. pylori foi erradicada apresentaram menor incidência de neoplasia de estômago, quando comparados aos que não receberam tal tratamento. Ressalta-se, no entanto, que literatura atual exibe resultados conflitantes, embora muitos estudos sugiram redução do risco por meio desta intervenção (SONNENBERG WR, 2017).

Além da infecção por $H$. pylori, alguns estudos apontam para a associação da neoplasia de estômago com o vírus Epstein-Barr (EBV), embora seu papel na patogênese permaneça desconhecido (SONNENBERG WR, 2017). O vírus em questão, pertencente à família dos herpesvírus, infecta 90 a $95 \%$ da população adulta mundial e encontra-se associado a $1 \%$ de todos os cânceres, com destaque especial aos linfomas de Hodgkin e Burkitt e carcinomas gástrico e nasofaríngeo (BAKKALCI D, et al., 2020). Alguns estudos estimam que a associação com este vírus é de aproximadamente $10 \%$, tornando o câncer de estômago a neoplasia mais comumente associada ao EBV (RUÍZ-GARGIA E, et al., 2017; BAKKALCI D, et al., 2020). 
Quadro 1 - Objetivos e principais resultados encontrados nos artigos selecionados.

\begin{tabular}{|c|c|c|}
\hline Autor & Objetivo de estudo & Resultados \\
\hline Bakkalci D, et al. (2020) & $\begin{array}{l}\text { Avaliar quais os fatores de risco que } \\
\text { contribuem para a ocorrência de cânceres } \\
\text { associados ao EBV e comparar os } \\
\text { resultados entre os tipos de neoplasias. }\end{array}$ & $\begin{array}{l}\text { - A etiologia dos cânceres associados ao EBV é complexa e } \\
\text { multifatorial, envolvendo características clínicas, genéticas e } \\
\text { ambientais. }\end{array}$ \\
\hline Balakrishnan M, et al. (2017) & $\begin{array}{l}\text { Discutir as tendências globais na incidência } \\
\text { do câncer de estômago e sua relação com } \\
\text { fatores de risco e patogênese da doença. }\end{array}$ & $\begin{array}{l}\text { - Redução global da incidência de devido à melhora } \\
\text { socioeconômica; } \\
\text { - Queda da prevalência de infecção por H. pylori reduziu a } \\
\text { incidência de câncer gástrico; } \\
\text { - Tabaco é fator de risco emergente; } \\
\text { - Pobreza associa-se à maior incidência. }\end{array}$ \\
\hline Braga L, et al. (2019) & $\begin{array}{l}\text { Avaliar a mortalidade por câncer de } \\
\text { estômago no Brasil no período de } 2000 \text { a } \\
2015 \text {. }\end{array}$ & $\begin{array}{l}\text { - O câncer gástrico ainda é uma grande causa de morbimortalidade } \\
\text { no Brasil; } \\
\text { - Região Nordeste, principalmente Ceará, é uma região de alto risco } \\
\text { para a doença. }\end{array}$ \\
\hline Cavatorta O, et al. (2018) & $\begin{array}{c}\text { Analisar a epidemiologia do câncer gástrico } \\
\text { e seus principais fatores de risco. }\end{array}$ & $\begin{array}{l}\text { - Fatores de risco modificáveis: tabaco, alcoolismo, radiação, } \\
\text { infecção por Helicobacter pylori; } \\
\text { - Fatores de risco não modificáveis: idade avançada, sexo } \\
\text { masculino, raça, história familiar de câncer gástrico, síndromes } \\
\text { genéticas. }\end{array}$ \\
\hline Chen P, et al. (2019) & $\begin{array}{c}\text { Determinar os fatores de risco } \\
\text { sociodemográficos e suas associações com } \\
\text { câncer gástrico. }\end{array}$ & $\begin{array}{l}\text { - Fatores de risco: baixo status socioeconômico, omissão do café- } \\
\text { da-manhã, vegetais em conserva, alimentação excessiva, não- } \\
\text { consumo de chá verde. }\end{array}$ \\
\hline Curado MP, et al. (2019) & $\begin{array}{c}\text { Descrever incidência, mortalidade e } \\
\text { tendências do câncer gástrico em São } \\
\text { Paulo, Belém e Fortaleza no período de } \\
1990 \text { a } 2012 .\end{array}$ & $\begin{array}{l}\text { - } \quad \text { Belém apresentou a maior incidência; } \\
\text { - Tendência à redução da incidência em São Paulo e Fortaleza; } \\
\text { - Tendência à redução da mortalidade por câncer gástrico em todas } \\
\text { as cidades avaliadas. }\end{array}$ \\
\hline Fang X, et al. (2015) & $\begin{array}{c}\text { Analisar a associação entre fatores } \\
\text { dietéticos e o risco de câncer de estômago. }\end{array}$ & $\begin{array}{l}\text { - Fatores de risco: alimentos com alto teor de sódio, cerveja, } \\
\text { destilados; } \\
\text { - Fatores protetores: frutas, vegetais brancos. }\end{array}$ \\
\hline
\end{tabular}




\begin{tabular}{|c|c|c|}
\hline Autor & Objetivo de estudo & Resultados \\
\hline Hirabayashi M, et al. (2019) & $\begin{array}{l}\text { Determinar a relação entre índice de massa } \\
\text { corpórea (IMC) e risco de câncer gástrico } \\
\text { através de um estudo de coorte prospectivo. }\end{array}$ & $\begin{array}{l}\text { - O risco de câncer gástrico aumenta em homens com IMC } \geq 27 \\
\mathrm{~kg} / \mathrm{m}^{2} \text {. }\end{array}$ \\
\hline Lee Y-C, et al. (2016) & $\begin{array}{c}\text { Investigar os efeitos da erradicação do } \\
\text { Helicobacter pylori na incidência de câncer } \\
\text { gástrico. }\end{array}$ & $\begin{array}{l}\text { - A erradicação do H. pylori associa-se à redução significativa da } \\
\text { incidência do câncer de estômago. }\end{array}$ \\
\hline Lope V, et al. (2016) & $\begin{array}{c}\text { Analisar a influência de fatores menstruais e } \\
\text { reprodutivos no risco dos cânceres gástrico } \\
\text { e colorretal. }\end{array}$ & $\begin{array}{l}\text { - Associação inversa entre idade no primeiro parto e risco de câncer } \\
\text { gástrico. } \\
\text { - Uso de anticoncepcional hormonal é fator protetor. } \\
\text { - Terapia de reposição hormonal pós-menopausa é fator protetor. }\end{array}$ \\
\hline Miao Z-F, et al. (2017) & $\begin{array}{l}\text { Avaliar as evidências que relacionam } \\
\text { diabetes mellitus à incidência e mortalidade } \\
\text { por câncer gástrico através da análise de } \\
\text { estudos de coorte. }\end{array}$ & $\begin{array}{l}\text { - Diabetes mellitus associou-se à maior incidência e mortalidade por } \\
\text { câncer gástrico, especialmente em homens e }<55 \text { anos. }\end{array}$ \\
\hline Mocellin S, et al. (2015) & $\begin{array}{l}\text { Analisar a associação entre variações do } \\
\text { DNA e o risco de câncer gástrico. }\end{array}$ & $\begin{array}{l}\text { - Diversos biomarcadores e polimorfismos associados a maior risco } \\
\text { de desenvolvimento de câncer gástrico foram identificados. }\end{array}$ \\
\hline Poorolajal J, et al. (2020) & $\begin{array}{l}\text { Determinar fatores de risco } \\
\text { comportamentais e dietéticos associados ao } \\
\text { risco de câncer de estômago. }\end{array}$ & $\begin{array}{l}\text { - Fatores de risco: infecção por } H \text {. pylori, tabagismo, etilismo, } \\
\text { sobrepeso/ obesidade, consumo de vegetais em conserva, } \\
\text { consumo de carne vermelha } \geq 4 X / \text { semana e alto consumo de sal; } \\
\text { - Fatores protetores: atividade física, consumo de frutas e vegetais } \\
\geq 3 X / \text { semana, consumo de chá verde, consumo de peixe } \geq 1 X / \\
\text { semana. }\end{array}$ \\
\hline Ruíz-García E, et al. (2017) & $\begin{array}{l}\text { Analisar os fatores de risco e alterações } \\
\text { moleculares associadas ao câncer gástrico. }\end{array}$ & $\begin{array}{l}\text { - Alta mortalidade por câncer gástrico na América Latina, devido ao } \\
\text { diagnóstico tardio; } \\
\text { - Fatores de risco: infecção por H. pylori e/ou EBV, tabaco, álcool, } \\
\text { consumo de carnes vermelhas e/ou processadas, dieta } \\
\text { hiperssódica, idade entre } 50 \text { e } 70 \text { anos. }\end{array}$ \\
\hline Salvador I, et al. (2015) & $\begin{array}{l}\text { Avaliar os fatores de risco e fatores } \\
\text { protetores para câncer/ metaplasia gástrica. }\end{array}$ & $\begin{array}{l}\text { - Fatores de risco: consumo de alimentos reaquecidos } \geq 3 X / \\
\text { semana, adição de sal em }>50 \% \text { dos alimentos. } \\
\text { - Fatores protetores: anti-inflamatórios não-esteroidais, idade }<58 \\
\text { anos, tratamento para H. pylori. }\end{array}$ \\
\hline
\end{tabular}




\begin{tabular}{|c|c|c|}
\hline Autor & Objetivo de estudo & - Resultados \\
\hline Sonnenberg W (2017) & $\begin{array}{l}\text { Discutir as características fundamentais das } \\
\text { principais malignidades gastrointestinais: } \\
\text { cânceres esofágico, gástrico e colorretal. }\end{array}$ & $\begin{array}{l}\text { - Tendência à queda da incidência de câncer gástrico nos Estados } \\
\text { Unidos (EUA), devido à melhoria das condições sanitárias; } \\
\text { - Não há screening para câncer de estômago nos EUA. }\end{array}$ \\
\hline Venerito M, et al. (2016) & $\begin{array}{l}\text { Analisar os principais aspectos clínicos e } \\
\text { epidemiológicos do câncer de estômago. }\end{array}$ & $\begin{array}{l}\text { - Linfoma de Hodgkin e anemia perniciosa relacionam-se à gastrite } \\
\text { - Autoimune e maior risco de câncer gástrico; } \\
\text { - Associação entre consumo de carne vermelha e câncer gástrico. }\end{array}$ \\
\hline Venerito M, et al. (2018) & $\begin{array}{l}\text { Avaliar a epidemiologia do câncer gástrico, } \\
\text { assim como suas estratégias de prevenção } \\
\text { e tratamento. }\end{array}$ & $\begin{array}{l}\text { - A erradicação do } H \text {. pylori reduziu significativamente o risco de } \\
\text { tumores metacrônicos em pacientes submetidos à ressecção } \\
\text { endoscópica de câncer gástrico precoce; } \\
\text { - Aspirina relacionou-se a menor risco de câncer de estômago; } \\
\text { - Inibidores de bomba de prótons possui papel controverso na } \\
\text { patogenia da doença em questão. }\end{array}$ \\
\hline Venneman K, et al. (2018) & $\begin{array}{c}\text { Analisar a prevalência da infecção por } H \text {. } \\
\text { pylori na Europa e determinar a influência } \\
\text { dos hábitos de vida na sua evolução para o } \\
\text { câncer gástrico. }\end{array}$ & $\begin{array}{l}\text { - Maior incidência de infecção por H. pylori na Europa Ocidental e } \\
\text { Sul, enquanto as menores taxas foram encontradas no Norte } \\
\text { Europeu; } \\
\text { - Fatores de risco: tabagismo, consumo de sal e carne vermelha; } \\
\text { - Fatores de proteção: consumo de frutas, vegetais e vitaminas; } \\
\text { - Fatores sem influência: consumo de álcool, atividade física. }\end{array}$ \\
\hline Wennerström ECM, et al. (2017) & $\begin{array}{l}\text { Determinar se a extensão proximal da } \\
\text { infecção por } H \text {. pylori decorrente da } \\
\text { supressão ácida implica em maior risco de } \\
\text { câncer de estômago proximal. }\end{array}$ & $\begin{array}{l}\text { - Exposição moderada a drogas supressoras de ácido do estômago } \\
\text { não se relacionou a maior risco de câncer gástrico proximal. }\end{array}$ \\
\hline
\end{tabular}

Fonte: Neves VH, et al., 2020. 
Os fatores dietéticos possuem grande papel na etiopatogenia do câncer de estômago. Em meta-análise de 76 estudos de coorte realizada por Fang X, et al. (2015), foi averiguado o impacto de múltiplos componentes dietéticos no risco de tal enfermidade. Constatou-se que o consumo de frutas e vegetais brancos é inversamente proporcional ao risco de neoplasias gástricas, visto que a vitamina $\mathrm{C}$, abundante em tais alimentos, não só reduz o dano oxidativo aos tecidos e à molécula de DNA, mas também inibe 0 crescimento e colonização pela $\mathrm{H}$. pylori, facilitando a penetração dos antibióticos na mucosa gástrica.

Ademais, os estudos de Fang X, et al. (2015) observaram ainda que a doença em questão se correlaciona positivamente ao consumo de insumos com alto teor de sódio e bebidas alcoólicas, principalmente destiladas. Enquanto o consumo de sal associa-se a processos irritativos da mucosa gástrica, gerando gastrite atrófica e alterações da síntese e proliferação celular, o álcool induz a formação de espécies reativas de oxigênio, considerados grandes mutagênicos. A ingesta de café, chá verde e outras bebidas, no entanto, não mostrou correlação significativa com o risco, embora haja resultados díspares na literatura.

Corroborando com as estatísticas supracitadas, a pesquisa de Venneman K, et al. (2018) associou o tabagismo, assim como o consumo de carnes processadas e alimentos ricos em sódio ao maior risco de câncer de estômago nos pacientes infectados por H. pylori. Em relação ao consumo de álcool, no entanto, o estudo em questão encontrou resultados colidentes. Venneman K, et al. (2018) ainda relacionou os tumores gástricos ao baixo status socioeconômico, alta densidade populacional e más condições de higiene. Outrossim, foi constatado que o consumo de frutas, vegetais e vitaminas acarretou em menor risco.

Poorolajal J, et al. (2020), através de revisão sistemática, verificou que, de fato, o principal fator de risco associado ao câncer gástrico é a infecção por $H$. pylori, seguida do tabagismo, que contribuiu para um aumento do risco em $61 \%$. Neste estudo ainda foi evidenciado que o etilismo aumenta o risco em $19 \%$ e 0 consumo de alimentos conservados em vinagre, em $28 \%$. Outrossim, o consumo de frutas e vegetais relacionou-se a redução do risco em até $62 \%$. Assim como em outras pesquisas, o consumo de café e chás verde e preto não influenciou o risco.

De modo a reforçar a influência dos fatores dietéticos na fisiopatogênese da doença, Salvador I, et al. (2015), em estudo caso-controle conduzido em hospital em Quito, no Equador, encontraram como fatores de risco o consumo de comidas reaquecidas $\geq 3$ vezes/ semana e a adição de sal em $>50 \%$ dos alimentos. Ademais, tal pesquisa determinou que o uso de anti-inflamatórios não esteroidais (AINEs), idade $<58$ anos e tratamento para a infeção por $H$. pylori, por sua vez, possuíam papel protetor no desenvolvimento de tal enfermidade.

No estudo de caso-controle conduzido por Chen P, et al. (2019) envolvendo 523 portares de câncer de estômago e 523 indivíduos saudáveis, foi verificado que os casos apresentavam maior prevalência de gastrite atrófica, menor escolaridade e status socioeconômico e maior consumo de alimentos quentes e de alto teor sódico, além de menor consumo de chá verde e tendência à maior ingesta alimentar e à omissão do café-damanhã. Em relação ao estado civil, observou-se que os controles eram mais frequentemente casados ou em união estável. Outrossim, problemas familiares e traumas psicológicos associaram-se positivamente à doença em questão.

Pesquisas ainda sugerem que o sobrepeso e a obesidade podem implicar em maior risco de neoplasia gástrica (HIRABAYASHI M, et al., 2019; SONNENBERG WR, 2017). Não obstante, salienta-se que diante da discrepância observada em múltiplos estudos, faz-se necessária a realização de pesquisas mais longas a fim de determinar com precisão esta relação (HIRABAYASHI M, et al., 2019).

Miao Z-F, et al. (2017), através da revisão de 22 estudos de coorte, constataram que os pacientes portadores de diabetes tiveram risco igual ou levemente aumentado para o desenvolvimento de câncer de estômago e morte por tal afeç̧ão, embora a literatura acerca da correlação entre tais condições clínicas traga resultados controversos.

O tratamento do linfoma de Hodgkin também já foi relacionado ao maior risco de neoplasias gastrointestinais, principalmente nos pacientes tratados com a combinação de irradiação infradiafragmática e quimioterapia contendo procarbazina, com índice de incidência padronizado (SIR) de 7,4\% (VENERITO M, et 
al., 2016). Outra condição considerada fator predisponente para a ocorrência de câncer de estômago, especialmente os não-cárdicos, é a anemia perniciosa, resultante de gastrite imunomediada (VENERITO M, et al., 2016).

O uso de determinados medicamentos também pode exercer papel relevante na etiopatogenia das neoplasias de estômago, como no estudo de caso-controle de Lope $\mathrm{V}$, et al. (2016), no qual verificou-se que as mulheres usuárias de contraceptivos hormonais apresentaram menor risco de desenvolver câncer gástrico e, embora a fisiopatologia envolvida nesta associação não seja completamente compreendida, acredita-se que esta guarde relação com o aumento da expressão do gene TTF, inibição da expressão de oncogenes e redução da concentração ácida da bile. Ressalta-se, no entanto, que meta-análises conduzidas recentemente não indicaram a associação supracitada. A exposição moderada a bloqueadores do receptor de histamina tipo-2 (H-2) ou inibidores da bomba de prótons (IBP), no entanto, não favoreceu o aparecimento de tumores proximais, de forma que a controvérsia existente na literatura acerca da contribuição destes medicamentos para a carcinogênese permanece (WENNERSTRÖM ECM, et al., 2017).

Embora as neoplasias gástricas sejam majoritariamente de caráter esporádico, 10\% dos casos encontramse associados a agrupamentos familiares (SONNENBERG WR, 2017) e, destas, em 1-3\% resultam de síndromes hereditárias, tais como o câncer gástrico difuso hereditário, polipose adenomatosa familiar, síndrome de Peutz-Jeghers e também alguns polimorfismos de nucleotídeo único, embora estes permaneçam controversos na literatura (CAVATORTA O, et al., 2018).

Mocellin S, et al. (2015), mediante revisão sistemática e meta-análise, identificaram 11 biomarcadores de alta qualidade relacionados à susceptibilidade ao câncer de estômago: MUC1 rs2070803, MTX1 rs2075570, PSCA rs2294008, PRKAA1 rs13361707, PLCE1 rs2274223, TGFBR2 rs3087465, PKLR rs3762272, PSCA rs2976392, GSTP1 rs1695, CASP8 rs3834129 e TNF rs1799724, além de outros polimorfismos genéticos, cada um deles associado alguma variante do tumor.

\section{CONSIDERAÇÕES FINAIS}

As neoplasias gástricas são afecções que, apesar de estar sofrendo mudança do seu perfil epidemiológico, com redução em sua incidência, ainda se constituem um grave problema de saúde pública, dado que é fonte de grande morbimortalidade. Esta afecção decorre da complexa interação entre fatores de risco ambientais, infecciosos e genéticos. Dentre os fatores de risco, os que se mostraram predominantes foram idade avançada, sexo masculino, infecção pela $H$. pylori, tabagismo, consumo de alimentos com alto teor de sódio e ingesta alcoólica. Outras condições também já foram implicadas, mas com menores evidências na literatura. Diante dos dados colhidos, conclui-se que o reconhecimento dos fatores de risco faz-se de suma importância para a prevenção e/ ou diagnóstico precoce dessa afecção, a fim de possibilitar melhora do seu perfil epidemiológico atual.

\section{REFERÊNCIAS}

1. BAKKALCI D, et al. Risk factors for Epstein Barr virus-associated cancers: a systematic review, critical appraisal, and mapping of the epidemiological evidence. Journal of Global Health, 2020; 10(1): 010405.

2. BALAKRISHNAN M, et al. Changing trends in stomach cancer throughout the World. Curr Gastroenterol Rep., 2017; 19(8): 36

3. BRAGA L, et al. Unequal burden of mortality from gastric cancer in Brazil and its regions, 2000-2015. Gastric Cancer, 2019; 22(4): 675-683.

4. CAVATORTA O, et al. Epidemiology of gastric cancer and risk factors. Acta Biomed, 2018; 89(8): 82-87.

5. CHEN P, et al. Risk factors of gastric cancer in high-risk region of China: a population-based case-control study. Asian Pacific Journal of Cancer Prevention, 2019; 20(3): 775-781.

6. CURADO MP, et al. Disparities in epidemiological profile of gastric adenocarcinoma in selected cities of Brazil. Asian Pacific Journal of Cancer Prevention, 2019; 20(8): 2253-2258.

7. FANG X, et al. Landscape of dietary factors associated with risk of gastric cancer: A systematic review and doseresponse meta-analysis of prospective cohort studies. European Journal of Cancer, 2015: 1-13.

8. HIRABAYASHI M, et al. Effect of body-mass index on the risk of gastric cancer: a population-based cohort study in a Japanese population. Cancer Epidemiology, 2019; 63: 101622. 
9. LEE Y-C, et al. Association between Helicobacter pylori eradication and gastric cancer incidence: a systematic review and meta-analysis. Gastroenterology, 2016; 150(5): 1113-1124.

10. LOPE V, et al. Menstrual and reproductive factors and risk of gastric and colorectal cancer in Spain. Public Library of Science, 2016; 10(11): 0164620.

11. MIAO Z-F, et al. Diabetes mellitus and the risk of gastric cancer: a meta-analysis of cohort studies. Oncotarget, 2017; 8(27): 44881-44892.

12. MOCELLIN S, et al. Genetic variation and gastric cancer risk: a field synopsis and meta-analysis. Gut, 2015; 64(8): 1209-1219.

13. POOROLAJAL J, et al. Risk factors for stomach cancer: a systematic review and meta-analysis. Epidemiology and Health, 2020; 42: 2020004.

14. RUÍZ-GARGIA E, et al. Gastric cancer in Latin America. Scandinavian Journal of Gastroenterology, 2017; 53(2):124129.

15. SALVADOR I, et al. Risk and protective factors for gastric metaplasia and cancer: a hospital-based case-control study in Ecuador. Nutrición Hospitalaria, 2015; 32(3): 1193-1199.

16. SONNENBERG WR. Gastrointestinal maligancies. Prim Care Clinics in Office Practice, 2017; 44(4):721-732.

17. VENERITO M, et al. Gastric cancer - clinical and epidemiological aspects. Wiley Online Library, $2016 ; 21(1): 39-44$.

18. VENERITO M, et al. Gastric cancer: epidemiology, prevention, and therapy. Wiley Online Library, 2018; 23(1): 12518.

19. VENNEMAN K, et al. The epidemiology of Helicobacter pylori infection in Europe and the impact of lifestyle on its natural evolution toward stomach cancer after infection: A systematic review. Wiley Online Library, 2018; 23(3):12483.

20. WENNERSTRÖM ECM, et al. Acid-suppressing therapies and subsite-specific risk of stomach cancer. British Journal of Cancer, 2017; 116(9):1234-1238. 